\title{
Estimation of microvascular perfusion after esophagectomy: a quantitative model of dynamic fluorescence imaging
}

\author{
Haryadi Prasetya ${ }^{1}$ (D) - Sanne M. Jansen ${ }^{1} \cdot$ Henk A. Marquering ${ }^{1,2} \cdot$ Ton G. van Leeuwen ${ }^{1} \cdot$ Suzanne S. Gisbertz ${ }^{3}$. \\ Daniel M. de Bruin ${ }^{1} \cdot$ Ed van Bavel $^{1}$
}

Received: 21 August 2018 / Accepted: 16 May 2019 / Published online: 26 June 2019

(C) The Author(s) 2019

\begin{abstract}
Most common complications of esophagectomy stem from a perfusion deficiency of the gastric conduit at the anastomosis. Fluorescent tracer imaging allows intraoperative visualization of tissue perfusion. Quantitative assessment of fluorescence dynamics has the potential to identify perfusion deficiency. We developed a perfusion model to analyze the relation between fluorescence dynamics and perfusion deficiency. The model divides the gastric conduit into two well-perfused and two anastomosed sites. Hemodynamics and tracer transport were modeled. We analyzed the value of relative time-to-threshold (RTT) as a predictor of the relative remaining flow (RRF). Intensity thresholds for RTT of $20 \%$ to $50 \%$ of the maximum fluorescence intensity of the well-perfused site were tested. The relation between RTT and RRF at the anastomosed sites was evaluated over large variations of vascular conductance and volume. The ability of RTT to distinguish between sufficient and impaired perfusion was analyzed using c-statistics. We found that RTT was a valuable estimate for low RRF. The threshold of $20 \%$ of the maximum fluorescence intensity provided the best prediction of impaired perfusion on the two anastomosed sites (AUC $=0.89$ and 0.86 ). The presented model showed that for low flows, relative time-to-threshold may be used to estimate perfusion deficiency.
\end{abstract}

Keywords Fluorescence imaging $\cdot$ Indocyanine green $\cdot$ Gastric conduit model $\cdot$ Perfusion $\cdot$ Esophagectomy

\section{Introduction}

Esophagectomy, despite the arduous nature of the procedure, is a commonly used surgical technique to treat esophageal cancer [1]. The procedure involves gastric transposition to the thorax and removal of major arterial and venous connections. Anastomotic leakage, necrosis, and stricture are major complications of this procedure. The success of esophagectomy depends on maintenance of perfusion of the whole gastric tube $[2,3]$. Particularly, in the fundus, i.e., the proximal end of

Haryadi Prasetya

h.prasetya@amc.uva.nl

1 Department of Biomedical Engineering \& Physics, Amsterdam University Medical Centers, Amsterdam, The Netherlands

2 Department of Radiology and Nuclear Medicine, Amsterdam University Medical Centers, Amsterdam, The Netherlands

3 Department of Surgery, Amsterdam University Medical Centers, Amsterdam, The Netherlands the gastric tube that is anastomosed with the remaining esophagus, perfusion is hampered as in this region, the perfusion fully depends on the presence of collateral connections. Insufficient perfusion hinders anastomotic healing or may even cause tissue necrosis. Early detection of insufficient perfusion could assist clinical decision making on additional surgical intervention, such as determination of the level of the anastomosis and primary anastomotic repair, consequently improving post-operative outcomes [4]. Accordingly, intraoperative monitoring of local perfusion in the gastric tube is needed to predict success of the procedure.

Recent developments in fluorescence imaging (FI) allow intra-operative visualization of local tissue perfusion in the gastric conduit [5-9]. This technique involves intravenous injection of indocyanine green (ICG) and monitoring of its appearance dynamics in gastric tissue. FI has shown a difference in fluorescence dynamics between native and anastomosed areas in the gastric conduit during surgery [4]. Preliminary experiments showed later time-to-peak of contrast arrival, suggesting lower perfusion, in areas closer to the fundus. However, the quantitative relation between contrast dynamics and actual perfusion of the gastric tissue has not been investigated. Moreover, 
alternative methods for detection of perfusion in this setting are not available.

Impaired perfusion results in slower contrast appearance compared to normal perfusion. Although time-to-peak is commonly used in dynamics measurements to assess perfusion, we hypothesize that time to intensity threshold is a valuable alternative because this measure can be performed in a shorter acquisition window of FI [4]. However, the validity of time to intensity threshold to assess local perfusion is unknown and may be complicated by the complexity of gastric tube vascular network, which includes collateral connections. In this study, we present a comprehensive model that describes the deteriorated perfusion after esophagectomy and its relation with temporal ICG fluorescence intensity profiles. This model was used to explore the relation between reduced perfusion and slower fluorescence enhancement at anastomosed areas. Because the volumes of these vascular compartments affect the dynamics $[10,11]$, the effect of variations in the vascular architecture of the gastric conduit on the relation between contrast dynamics and local perfusion was studied as well. We included a wide range of vascular resistances and volumes in order to identify general trends in ICG enhancement dynamics as a measure for perfusion. Finally, those trends were used to evaluate the usefulness of time to intensity threshold as an estimate for local perfusion.

\section{Methods}

Essentially, we defined a simulation model of the gastric tube that includes perfusion and ICG transport in four regions. Local perfusion in this model was determined for a large range of model parameters. RRF, the relative remaining flow, is the calculated flow after the intervention relative to the flow to that compartment before the intervention and is considered to be a predictor of clinical outcome. RTT, the relative time to threshold, is the calculated time to a threshold signal for local ICG appearance, normalized to the time to threshold in the first, well-perfused compartment. RTT is considered to be a surrogate for RRF. We analyzed how well RTT predicts RFF, how this depends on the chosen parameters, and which threshold should be taken.

\subsection{Gastric conduit model}

The reconstruction of the gastric conduit generally preserves the right gastroepiploic vessels and right gastric artery as the main source of blood supply to the gastric wall. Consequently, part of the gastric conduit close to the anastomosis is only supplied with blood from collateral connections in the gastric wall. We modeled the gastric conduit by introducing four sites, as shown in Fig. 1. This choice was based on the four regions of interest (ROIs) for measurement of fluorescence intensity performed on constructed gastric conduit in the prospective clinical study in
Amsterdam Medical Center from October 2015 to June 2016 [12]. Figure 1a shows a frame of constructed gastric conduit of a patient with the ICG visualizing the tissue perfusion. The ROI was a 300 pixels circle with \#1 $3 \mathrm{~cm}$ below the watershed, \#2 the watershed, \#3 $3 \mathrm{~cm}$ above the watershed, and \#4 the fundus. The measured fluorescence enhancement curves at the four ROIS are shown in Fig. 1b. In the model, the four sites are connected through collateral arteries and veins. Site 3 and site 4 represent the anastomosed areas and perfusion here depends completely on collateral vessels. The distributed nature of the arterial, capillary, and venous networks at each site is represented by a single lumped resistance thought to be situated in the arterioles and capillaries, connecting a proximal arterial volume to a distal capillary/venous volume. The vascular bed in the gastric tube is thus represented by eight compartments, four microvascular connections, three arterial collateral connections, and three venous collateral connections. The system is supplied and drained by large arteries and veins of the lower two sites (Fig. 1c).

\subsection{Parameter space of the model}

We assumed identical vascular conductances and volume for each site and varied multiple model parameters including collateral artery and vein conductance relative to microvascular conductance $\left(G_{c a} / G_{c}\right.$ and $\left.G_{c v} / G_{c}\right)$, relative large vessel conductance $\left(G_{L V} / G_{c}\right)$, and vascular volume including arterial and venous volume ( $A V$ and $V V$ ). Quantitative information on gastric vascular branching patterns is not available, but we considered that the above conductance ratios are likely to be highly variable between patients. We therefore evaluated the model over a large parameter space, including the presumed physiological range. Determining the physiological range for vessel conductance is difficult. Total conductance of the arterial and venous system depends on the network connectivity and the diameter of the individual segments in this network. Such data are, to the best of our knowledge, not available for the human stomach. We therefore covered a very wide range of values for $G_{c a} / G_{c}$ and $G_{c v} / G_{c}$, spanning 0.01 to 100 for both ratios. The low end of the chosen spectrum reflects absence of collaterals, while the high end indicates a model with identical perfusion of all four sites. Physiological values were estimated to be less than 10 , with no definitive lower boundary $[13,14]$. The large vessels (LV) include terminal arteries, i.e., right gastric artery and right gastroepiploic terminal artery, and initial veins, i.e., pyloric vein and right gastroepiploic initial vein. Relative conductance of the combined large arteries $\left(G_{L V} / G_{c}\right)$ was 3 by default and spanned 1 to 100 when studying the effect of this parameter. Large vein conductance was fixed at twice the large artery conductance. We used physiological data describing the proportion of artery, capillary, and vein in a given volume in the systemic circulation to determine the physiological vascular volumes $[14,15]$. The total volume of a site was calculated based on the volume of the ROI in the preceding clinical study [12]. The default vascular volumes were taken as $0.24 \mathrm{~mL}$ and 
Fig. 1 An image of constructed gastric conduit (a), obtained intraoperatively during fluorescence imaging postesophagectomy, demonstrated perfusion with ICG and reduced fluorescence in the collateraldependent upper part. The corresponding fluorescence enhancement curves of each ROI were measured at the gastric conduit (b). The model (c): from bottom to top: the sites $1-4$, which correspond, respectively, to the ROIs. A site consists of an arterial and a venous compartment directly connected through a capillary bed (horizontal line between compartments). Collaterals, as depicted by vertical lines between compartments, connect the adjacent sites. In this figure, sites 1 and 2 are well perfused, whereas sites 3 and 4 are anastomosed. Arrows indicate direction of flow after the intervention

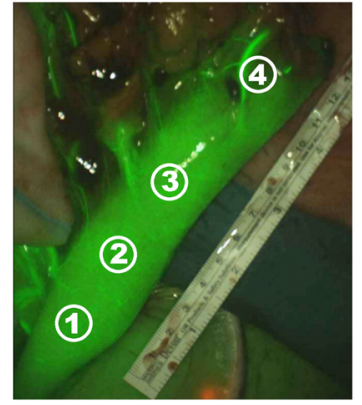

a

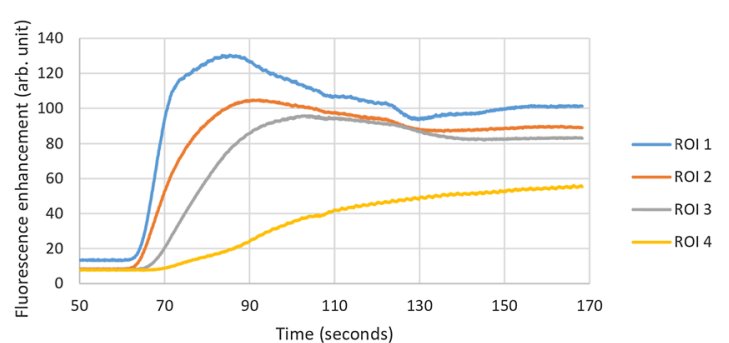

b

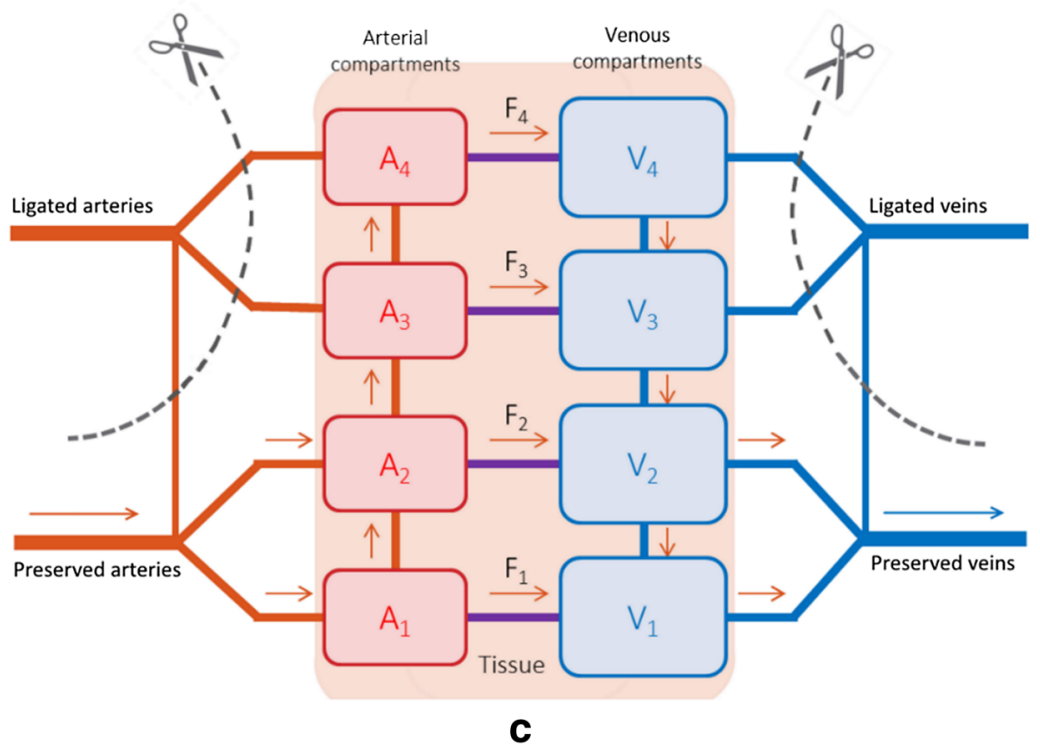

$0.56 \mathrm{~mL}$ for arterial $(A V)$ and venous $(V V)$ compartments, respectively, spanning $0.12 \mathrm{~mL}$ to $1.12 \mathrm{~mL}$ when studying the effect of vascular volume to contrast dynamics.

\subsection{ICG transport simulation}

Pressure at each branching point in Fig. 1c and flow in each segment was calculated using Kirchhoff's first law combined with Ohm's law, assuming laminar flow of a Newtonian fluid [16]. These calculated hemodynamic parameters depend on the pressures in the right gastroepiploic artery and vein and on the conductance of all segments. Arterial input and venous outflow pressure were taken as $70 \mathrm{mmHg}$ and 0 , respectively. Conductances were varied to evaluate their influence on perfusion. For each segment, after the pressure gradient was obtained, the flow was calculated. Tissue perfusion at the four sites is reflected by the predicted flow in the segments connecting arterial and venous compartments $\left(F_{1}\right.$ to $\left.F_{4}\right)$.

The ICG enters the system from the hepatic artery into the gastroduodenal artery (greater curvature), which leads into right gastroepiploic artery, and the right gastric artery (lesser curvature). The ICG then flows into the various compartments from $A_{1}$ and $A_{2}$ towards capillaries in the native sites and collateral-dependent sites and leaves the system via $V_{l}$ and $V_{2}$. ICG from the right gastric and the right gastroepiploic veins drains into the portal and the superior mesenteric vein, respectively. This ICG transport was simulated using the above vessel configuration and well-mixed arterial and venous compartments, where dynamics of dye concentration obey the following differential equation:

$\frac{d C_{k}}{d t}=\frac{\sum_{j=1}^{m} U C_{j} \cdot U F_{j}-C_{k} \sum_{i=1}^{n} D F_{i}}{V_{k}}$

with $C_{k}$ the concentration of the dye in compartment $k, m$, and $n$ the number of upstream and downstream vessel of compartment $k$, respectively, $U C_{j}$ the concentration of upstream compartment $\mathrm{j}, U F_{j}$ upstream flow from this compartment, $D F_{i}$ flow through $i$-th downstream vessel, and $V_{k}$ the volume of compartment $k$ [17]. This ordinary differential equation was numerically solved with a single-step solver based on the Dormand-Prince algorithm of Runge-Kutta method which computed $C(t)$ from $[C(t-\Delta t)][18]$. The algorithm had six stages of function evaluation for each partial step and 
Fig. 2 Example of contrast dynamics at the four sites with derivation of the respective times to threshold. Sites 1 and 2 have native perfusion, while sites 3 and 4 depend on collateral flow, and accordingly have slower contrast dynamics

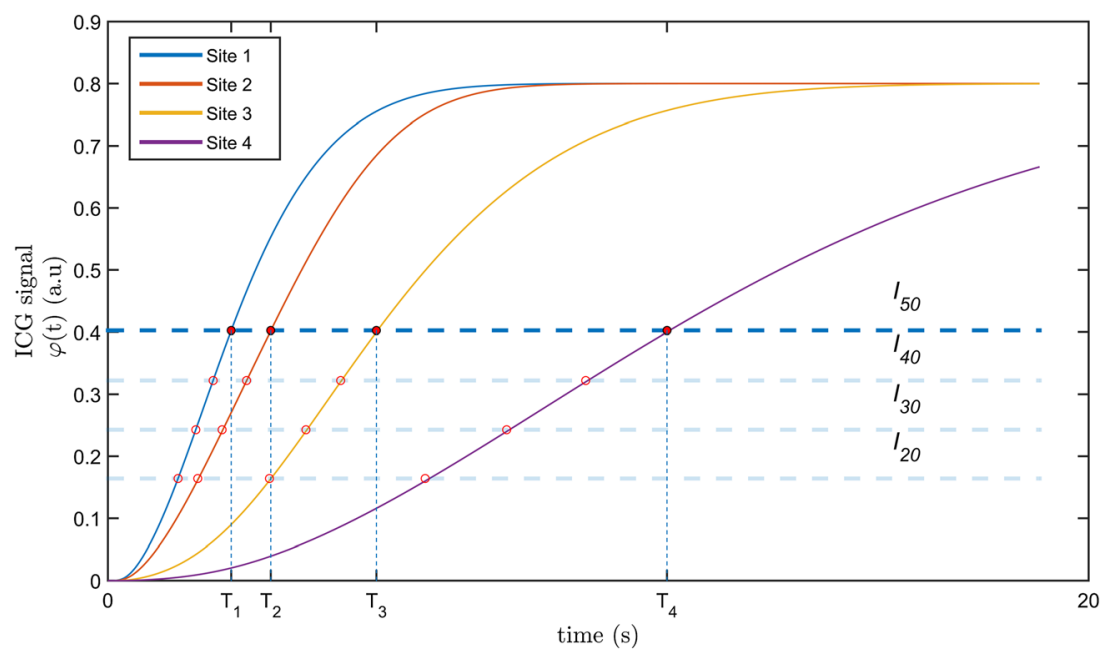

generated fourth-order and fifth-order approximation of $C(t)$. The two approximations was subsequently compared to estimate the error which provides the basis to accept or reject the tentative $C(t)$. The error estimate also modulated the step size, $\Delta t$, for the next time step.

The ICG signal intensity for each site was defined as the amount of ICG in both the arterial and venous compartments at time $t$ :

Table 1 Parameters of the model, simulation scenarios, and generated variables
$\varphi_{i}(t)=A C_{i}(t) \cdot A V_{i}+V C_{i}(t) \cdot V V_{i}$

where $\varphi$ indicates the ICG signal, $A C$ and $A V$ denote the ICG concentration and the volume of the arterial compartment, respectively. $V C$ and $V V$ represent the ICG concentration and the volume of the venous compartment and $i$ denotes the number of site. Figure 2 shows an example of the simulated temporal profile of $\varphi$ for each site.

\begin{tabular}{lllll}
\hline Parameters & Definitions [unit] & Default & Min & Max \\
\hline$G_{c a} / G_{c}$ & relative collateral artery conductance [-] & & 0.01 & 100 \\
$G_{c V} / G_{c}$ & relative collateral vein conductance [-] & & 0.01 & 100 \\
$G_{L V} / G_{c}$ & relative large vessel conductance [-] & 3 & 1 & 100 \\
$A V$ & arterial volume [ml] & 0.24 & 0.12 & 1.12 \\
$V V$ & venous volume [ml] & 0.56 & 0.12 & 1.12
\end{tabular}

Simulation 1

Model parameters varied: $G_{c a} / G_{c}, G_{c v} / G_{c}$

Model parameters fixed to default: $G_{L V} / G_{c}, A V, V V$

Simulation 2

Model parameters varied: $G_{c a} / G_{c}, G_{c v} / G_{c}, G_{L V} / G_{c}$

Model parameters fixed to default: $A V, V V$

Simulation 3

Model parameters varied: $G_{c a} / G_{c}, G_{c v} / G_{c}, A V, V V$

Model parameters fixed to default: $G_{L V} / G_{c}$

Generated variables

Solving Kirchhoff's first law + Ohm's law $F_{1}, F_{2}, F_{3}, F_{4}$

Solving eq.1 \& eq.2 $\quad T_{1}, T_{2}, T_{3}, T_{4}$; for threshold $20 \%$ of maximum $\varphi$ of site $1\left(I_{20}\right)$

$T_{1}, T_{2}, T_{3}, T_{4}$; for threshold $30 \%$ of maximum $\varphi$ of site $1\left(I_{30}\right)$

$T_{1}, T_{2}, T_{3}, T_{4}$; for threshold $40 \%$ of maximum $\varphi$ of site $1\left(I_{40}\right)$

$T_{1}, T_{2}, T_{3}, T_{4}$; for threshold $50 \%$ of maximum $\varphi$ of site $1\left(I_{50}\right)$

${ }^{*} F_{\text {presurgery }}$ were similar across the sites 


\subsection{Time-based perfusion estimate}

For each simulation, the temporal profile of $\varphi$ at each site was analyzed. This signal forms the base for the estimation of remaining perfusion after surgery. We propose a measure to describe relative impairment of flow in a collateral-dependent site. This measure was based on the time to threshold $T_{n}$ which was defined as the time at which $\varphi$ of site $n$ reaches a fraction of the maximum of $\varphi$ of site 1. For example, in Fig. 2, $T_{4}$ is defined as the time it takes for $\varphi$ to reach $50 \%$ of the maximum intensity in site $1\left(I_{50}\right)$. This approach ensures that $T_{\mathrm{n}}$ can be calculated as long as the maximum $\varphi$ in site 1 is recorded. We introduce RTT as a parameter on which RRF estimation can be based:

$$
\begin{array}{ll}
R T T_{3}=T_{1} / T_{3} & R T T_{4}=T_{1} / T_{4} \\
R R F_{3}=F_{3} / F_{3 \text { presurgery }} & R R F_{4}=F_{4} / F_{4 \text { presurgery }}
\end{array}
$$

With $T_{i}$ the time to threshold and $F_{i}$ the flow in site $i . F_{\text {presurgery }}$ is the flow prior to the ligation. It was calculated from the same model and using the same set of parameter values but with $A_{3}$
Fig. 3 Result of simulation 1: relation between RTT and RRF over collateral conductance space, for sites 3 (left) and 4 (right). The plots show RTT for thresholds of contrast arrival at $20 \%$ (a), $30 \%$ (b), $40 \%$ (c), and $50 \%$ (d) of the maximum contrast signal at site 1 as a function of RRF. The corresponded fitting functions and the respective goodness-of-fit parameters were also displayed a $\operatorname{RRF}$ vs $\operatorname{RTT}\left(I_{20}\right)$

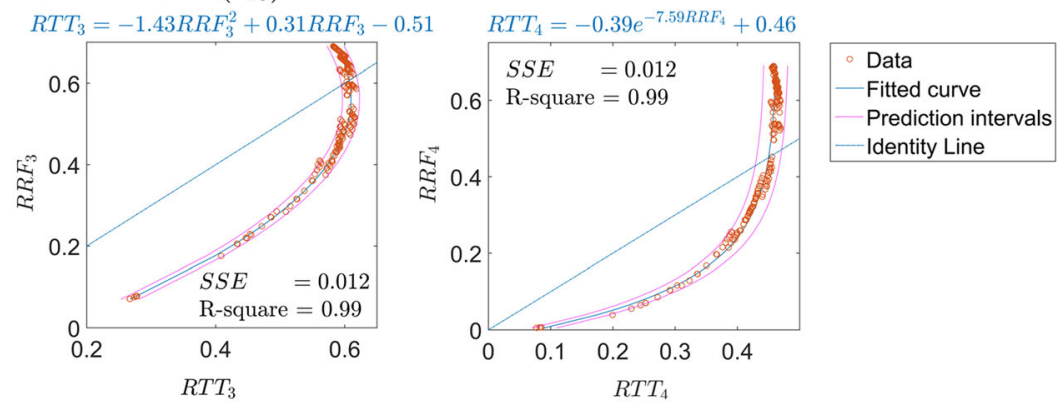

b $\operatorname{RRF}$ vs $\operatorname{RTT}\left(I_{30}\right)$
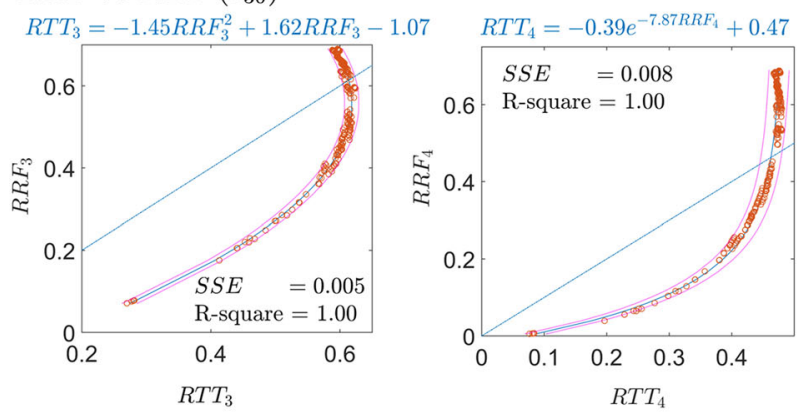

c RRF vs RTT $\left(I_{40}\right)$
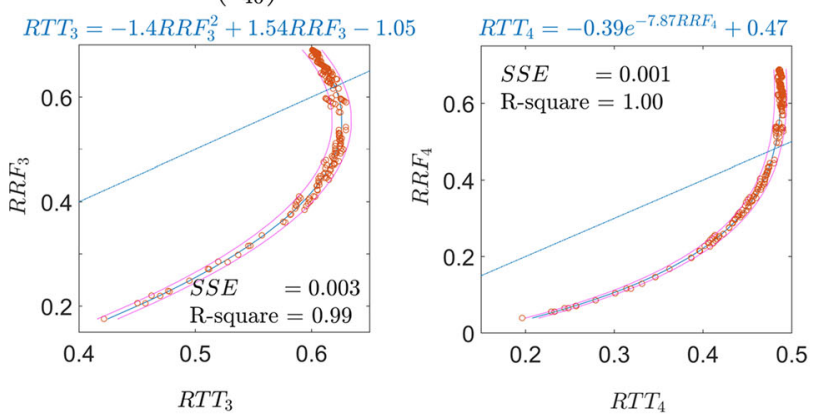

d $\operatorname{RRF}$ vs $\operatorname{RTT}\left(I_{50}\right)$
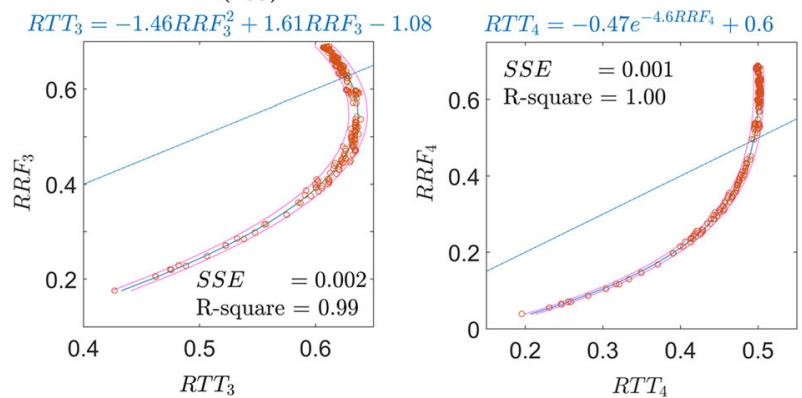
Fig. 4 Result of simulation 2: variation in large vessels conductance changes the relation between true RRF and its estimate (RTT)

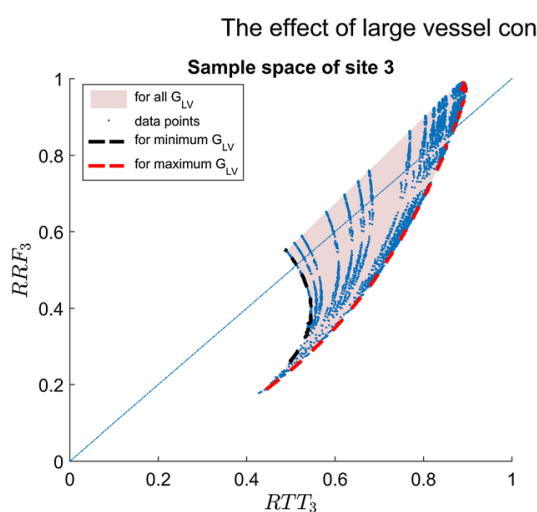

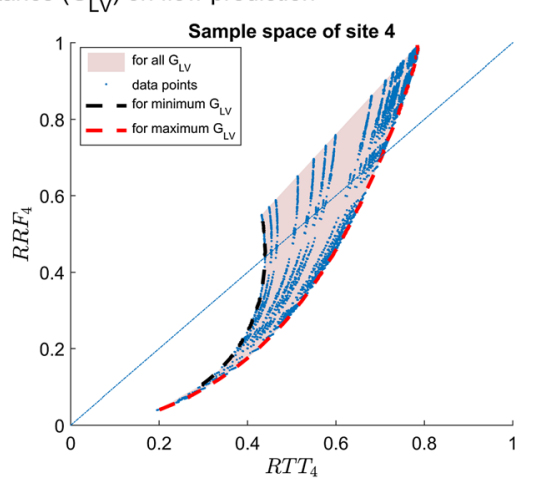

and $A_{4}$ still supplied by the large arteries and $V_{3}$ and $V_{4}$ still drained by the large veins. A range of intensity thresholds were tested: $20 \%, 30 \%, 40 \%$, and $50 \%$ of the maximum $\varphi$ of site 1 . The starting point $(t=0)$ was defined as the first inflection of contrast intensity in site 1 . RRF is the ratio of post- and preintervention flow. We hypothesized that for a large range of variation in vascular conductances and volumes, RTT is closely related to RRF, such that RTT is a possible measure for the effect of surgery on local perfusion.

Using the simulation scenarios detailed in the Table 1, we first evaluated the effect of $G_{c a} / G_{c}$ and $G_{c v} / G_{c}$ on the remaining perfusion in anastomosed sites by fixing $G_{L V} / G_{c}, A V$ and $V V$ to their default values (simulation 1). The effect of $G_{L V} / G_{c}$ was tested while fixing $A V$ and $V V$ to 0.24 and $0.56 \mathrm{ml}$, respectively (simulation 2). Finally, $G_{L V} / G_{c}$ was maintained at its default value (3) while examining the effect of $A V$ and $V V$ (simulation 3). All tests were performed using all intensity thresholds over the full range of collateral conductances. We then computed the RRF for all possible combinations of parameters for a range of possible outcomes (sample space) from Ohm's and Kirchhoff's laws. We included curve fits of the relation between RRF and RTT to illustrate the nature of the relation over collateral conductance space. We included curve fits of the relation between RRF and RTT to illustrate the nature of the relation over collateral conductance space. We tested the curve fit using linear, polynomial, and exponential function while evaluating the goodness-of-fit. A good fit was defined as a model that has low sum of squared of errors and high $R^{2}$. The prediction interval was calculated by taking into account the sample mean, sample standard deviation, sample size, and critical value of Student's $t$ distribution at $95 \%$ confidence level. It should be noted that the indicated relations merely described the data and were not a result of mathematical analysis of the model. Hence, these fits do not affect the further outcomes in this study. Finally, we performed receiver operating characteristic (ROC) curve analysis to select the best intensity threshold to estimate perfusion impairment. We arbitrarily chose RRF values to dichotomize the outcome into lacking versus adequate perfusion. For site $3, \mathrm{RRF}<50 \%$ was defined as lacking perfusion, and $\mathrm{RRF} \geq 50 \%$ was adequate perfusion. For site $4, \mathrm{RRF}<40 \%$ was defined as lacking perfusion, and $\mathrm{RRF} \geq 40 \%$ was adequate perfusion. The area of physiological sample space was treated as predictor variables on which logistic regression was applied to produce the ROC curve. The simulation was performed in Matlab on a standard PC running on Windows 7 with $3 \mathrm{GHz} 16 \mathrm{CPUs}$, 32 GB RAM, and NVIDIA Quadro K4200 GPU.

\section{Results}

The relation between RRF and RTT for sites 3 and 4 is shown in Fig. 3, where the estimates are based on time to reach $20 \%$ (Fig. 3a) to $50 \%$ (Fig. 3d) of the maximum $\varphi$. The increase in
Fig. 5 Result of simulation 3: RRF and RTT relation as a function of the ratio of arterial volume $(A V)$ and venous volume $(V V)$. Note that not every symbol/ color is visible due to the overlay

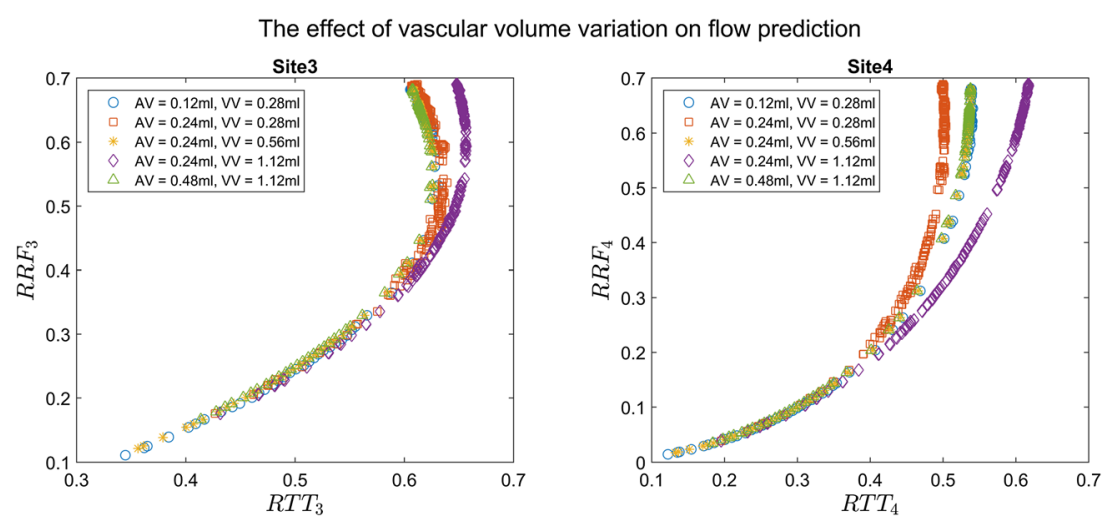


collateral conductance correlates with higher RTT and RRF. Interestingly, aside from negligible variation in the resulting RRF, the varying balance of conductance between collateral arteries and collateral veins seems to be inconsequential (see Appendix). As can be seen, the relation between RTT and RRF is far from linear, with RRF being lower than RTT for low flows. For high flows, RTT becomes stable and insensitive to flow changes. We obtained good fits of the relation at site 3 by second-order polynomials, while for site 4 , an exponential fit was needed. The RTT-RRF relation for various values of $G_{L V}$ is illustrated in Fig. 4. For low values of $G_{L \text { b }}$ the large vessels limit perfusion, thereby reducing the maximum possible levels of RRF. Figure 5 shows the RTT-RRF relation for various vascular volumes. The RTT-RRF relation for low flows is relatively independent of the volumes. However, for higher flows, i.e., better collaterals, the volumes indeed have an influence but rather than the absolute volume values, it is the ratio of arterial and venous volume that matters.

Reference image: determining RRF through RTT
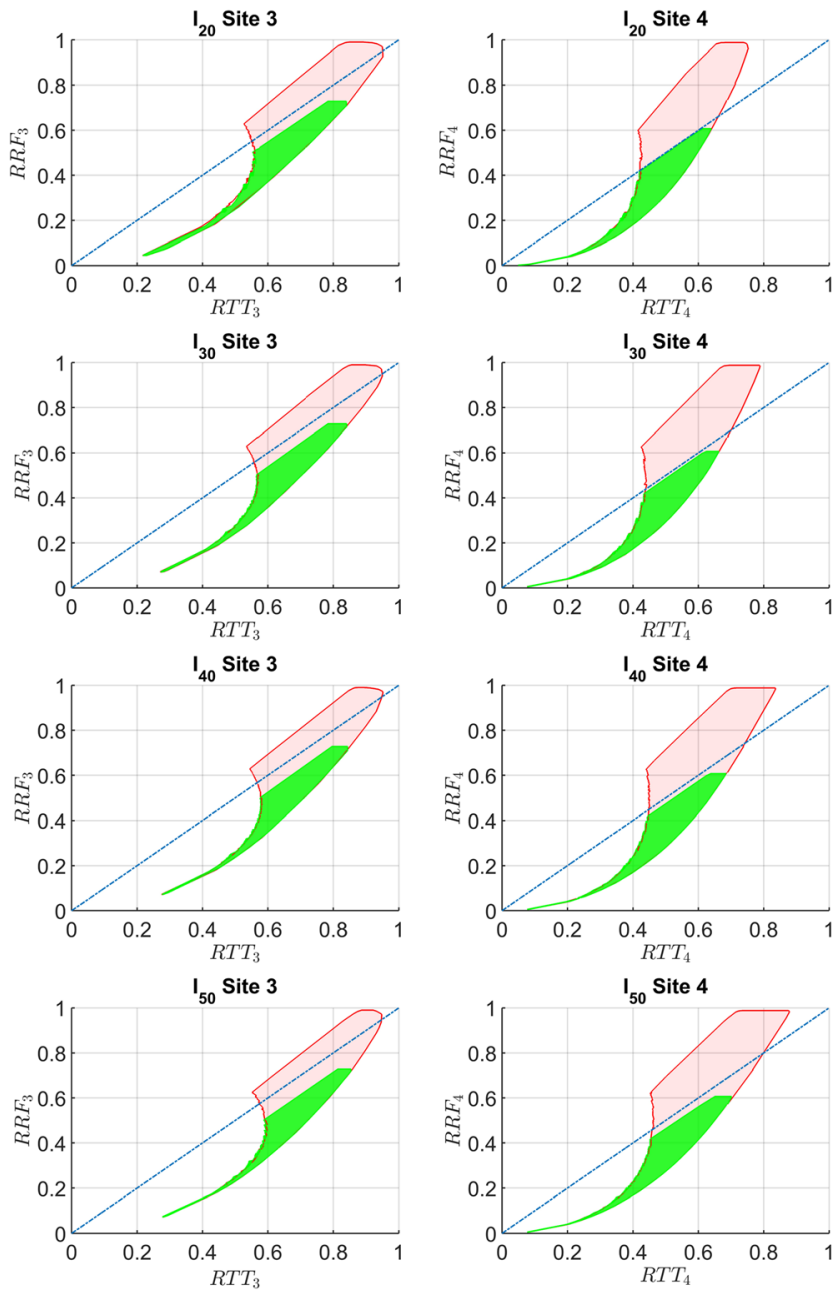

Fig. 6 The sample space of the model from the complete (pink) and physiological (green) parameter space of collateral conductances $\left(G_{c a}\right.$, $\left.G_{c v}\right)$, large vessel conductance $\left(G_{L V}\right)$, and vascular volume $(A V, V V)$ was calculated from $I_{20}$ to $I_{50}$ (top to bottom)
Figure 6 shows the relation between RTT and RRF for all above variations in conductances and volumes (4 sets of parameters). The pink area indicates the sample space for the full range of variations, while the green area denotes possible outcomes when the parameters were varied between more realistic values.

The highest accuracy of RTT as RRF estimator resides in both extrema of RTT, particularly in the lower values. As example, for an observed RTT with $I_{20}$ at site 4 of 0.2 , the true RRF may have been 0.038 to 0.04 , as compared to RTT of 0.5 which may have resulted from a larger range of true RRF from 0.31 to 0.5 . Assessed by ROC, all four logistic regression models demonstrated good discriminatory capacity in both sites (see Fig. 7). Although all intensity thresholds performed comparably well, $I_{20}$ yielded the highest concordance of predictions with actual outcomes (AUC $=0.89$ and 0.86 for sites 3 and 4 , respectively).

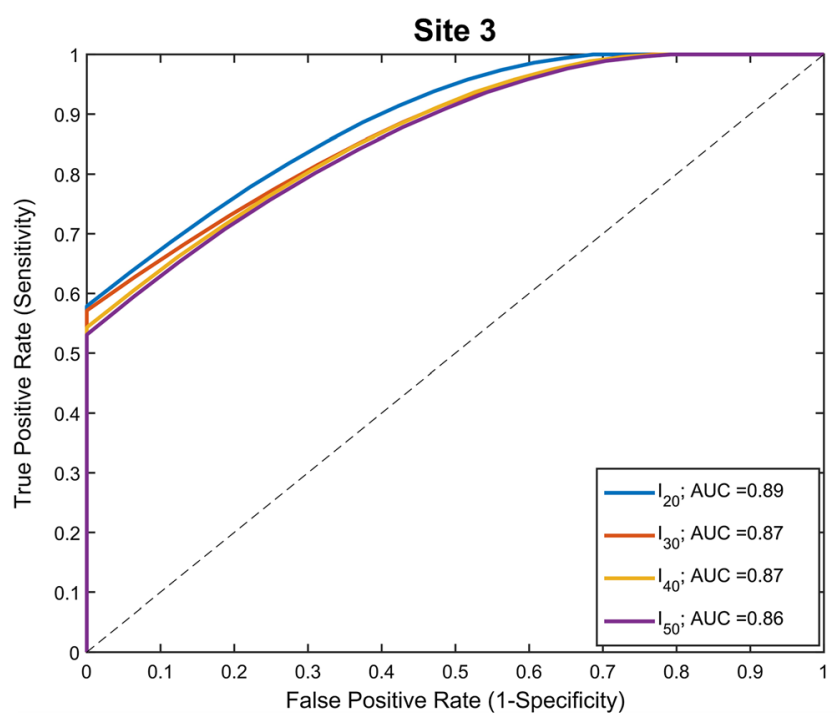

Site 4

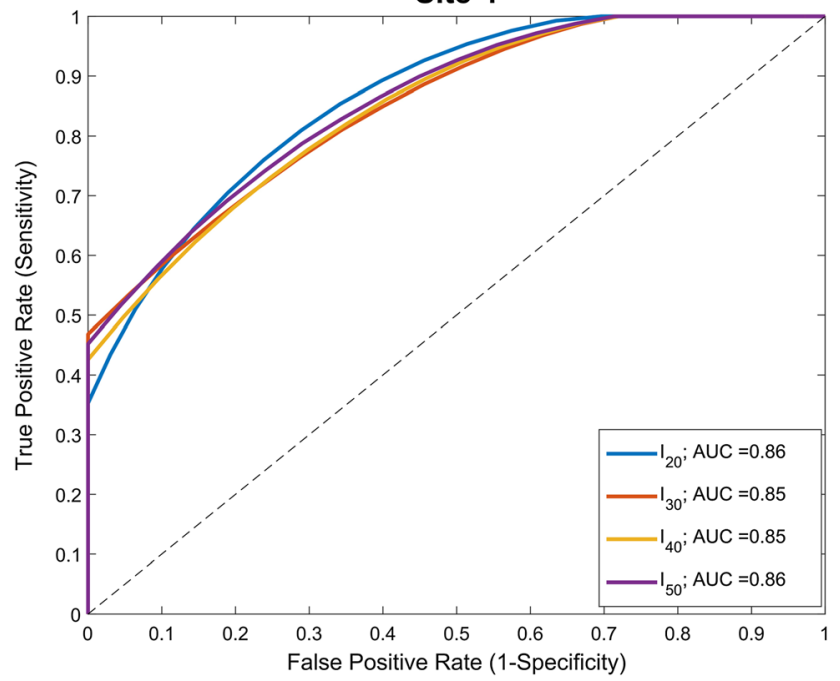

Fig. 7 ROC curve of four threshold for site 3 (top) and 4 (bottom). The cutoff value was determined hypothetically as 0.5 and 0.4 for sites 3 and 4 , respectively 


\section{Discussion}

In this study, we introduced a gastric conduit model and used this to identify the effects of various combinations of vascular conductance and volume on ICG dynamics. The results indicate that the relation between delayed time-to-threshold (indicated by RTT) and reduced flow (indicated by RRF) is not trivial. We have shown a strong dependency of this relation on collateral conductance, large vessel conductance, and vascular volume. Despite this dependency, we found that time-tothreshold at the collateral-dependent sites can be used to estimate whether remaining perfusion is sufficient.

\subsection{Perfusion model analysis}

The vascular tree can be modeled using several choices for the level of detail of the branching network. One extreme is to have a full network of vessel segments, with poroelastic models for the smallest vessels [19-21]. The other extreme would be a singlevessel lumped model that disregards the detailed geometric structure of vascular tree thus reducing the complexity of the model $[10,22,23]$. We believe that the current choice for a limited set of compartments is optimal for the current purpose since the actual small vessel structure in the ROI of the gastric conduit is not well-defined and likely to be highly variable. The four ROIs used in this study, which account for both arterial and venous volumes, are based on a preliminary study using clinical data [12]. The ROI 1, 2, and 3 were equidistant ( $3 \mathrm{~cm}$ apart), while ROI 4 was located in the fundus. We separated arterial and venous network into two compartments of lumped vessels since these compartments influence the contrast dynamics in each ROI. Hence, this model allowed us to evaluate the role of the arterial and venous collateral on the tissue perfusion. Other configuration of the model was not investigated (for instance an eight-site model). This choice would affect the values of the modeling parameters, since clearly the volumes and conductances in each site would be different. Yet, there seems little reason to suspect that the modeling outcomes and further analysis would be fundamentally different for such a more detailed model. Considering the clinical setting we therefore used the four site model.

\subsection{Limitations}

The validity of the presented model remains a limitation as this study only uses hypothetical data. We partly addressed this limitation by varying several model parameters over large ranges. Yet, many other choices would have been possible. Thus, the assumption of identical collateral conductances for all sites is an oversimplification of real cases. Patient data also show high variability in vascular volume between sites. These variations may yield different relations between RTT and RRF. The accuracy of the predictive value of RTT to predict RRF was based on perfusion thresholds of $50 \%$ and $40 \%$ of the pre- intervention perfusion. Currently, we do not have data for realistic values of this threshold.

A strong point of this study is that we have created a model in which many parameters can be tested. Therefore, alternative measures (such as intensity based, or absolute times) for estimating the perfusion can be evaluated with this model. However, in the context of the complex surgery, observation periods are limited to 2-3 min and longer periods are a concern [24]. In the fluorescence enhancement curve in Fig. 1b, the fluorescence yield is reasonably similar between the sites and a maximum is obtained in also the fourth site but these are not always the case. Thus, alternative measures of perfusion that rely on normalization to the maximal fluorescence at site 4 or generally the full enhancement curve may have been impractical.

\subsection{Potential use of gastric conduit model}

A large number of studies have employed fluorescence imaging in intraoperative applications to assist visualization of blood flow or anatomical features [6, 25-28]. However, quantitative analysis of ICG fluorescence imaging of the gastric conduit is still limited. Yukaya et al. introduced a quantitative parameter describing the decay of luminance as analyzed with the software tool LumiView to predict anastomotic leakage [29]. They could not find an association between blood flow and anastomotic leakage. A study on quantitative assessment of free jejunal graft used the time-fluorescence intensity curve, showing that time to half maximum is an indicative parameter for venous malperfusion [30]. However, that study had a population of only five patients suffering from venous anastomotic failure. Furthermore, in that study, no direct relation between perfusion deficit and ICG intensity dynamics was studied. A more recent study had been performed to predict anastomotic leakage by quantitatively measuring ICG speed between four predetermined points in the gastric conduit [8]. Also, this study suffered from limited data especially in the anastomotic leakage/malperfusion group.

While there clearly are several limitations to consider, quantitative analysis of contrast dynamics could provide a useful prognostic tool in determining treatment success. We found that time to $20 \%$ of the maximum intensity is optimal in the discrimination between intermediate and low perfusion as indicated by the area under the ROC curve. Additionally, if adopted in clinical practice, this low threshold requires only a relatively short measurement time of fluorescence imaging after maximum intensity is reached in ROI 1, which alleviates surgery-related risks.

Since fluorescence imaging allows assessment of temporal ICG intensity for different areas of gastric conduit intraoperatively, the operating clinician can evaluate intensity profiles for selected ROIs. This allows the calculation of the RTT at the anastomosed site and this value can be used to estimate the range of perfusion reduction (Fig. 6). The actual usefulness of RTT as an 
estimate for local perfusion and predictor of final outcome in esophagectomy remains to be established.

\section{Conclusion}

Our model demonstrated the effects of vascular conductance and volume on contrast dynamics in gastric conduit in relation to perfusion in anastomosed areas. After evaluating ICG dynamics for numerous different model parameters, we found that the relation between the dynamics and perfusion is not trivial. However, the model showed that for low flows, a low time to threshold intensity is predictive of flow deterioration. This estimation of remaining perfusion may form the base for clinical evaluation of a successful esophagectomy.

Funding information The work presented in this article was supported by the Indonesia Endowment Fund for Education (LPDP), Ministry of Finance, Republic of Indonesia through LPDP Scholarship Program for Doctorate Students and by the grant from The Netherlands Organization for Health Research and Development (ZonMw project\# 104002006).

\section{Compliance with ethical standards}

Conflict of interest Henk A. Marquering is a co-founder and shareholder of Nico.lab. Ton G. van Leeuwen reports grants from ZonMW.
Suzanne S. Gisbertz reports a consultancy agreement with Medtronic and grants from Olympus outside the submitted work. The other authors declare that they have no conflict of interest.

\section{Appendix}

The effect of collateral artery and vein conductance ratio and total collateral conductance to capillary perfusion

The balance ratio between collateral artery and vein conductance $\left(G_{c a} / G_{c v}\right)$ reflects the contribution of one side of collateral over another in determining perfusion. We compared the RRF modeled with a specific collateral conductance ratio with its inverse to test whether a certain side of collaterals is more favorable for perfusion. The result has shown negligible difference between RRF of two conductance configuration. Eventually, it was apparent that the total collateral conductance is a more consequential factor in determining the portion of contrast flowing through the capillary. Total collateral conductance was calculated as the inverse of the sum of collateral arteries and veins resistance in a series circuit $\left(G_{c a}\right.$. $\left.G_{c v} /\left(G_{c a}+G_{c v}\right)\right)$. Figure 8 shows the effect of this total collateral conductance in logarithmic scale on relative remaining flow in these models. The ratio of arterial and venous conductance has a relatively minor impact on these perfusions while increasing total collateral conductances show a better perfusion of the collateral-dependent sites.

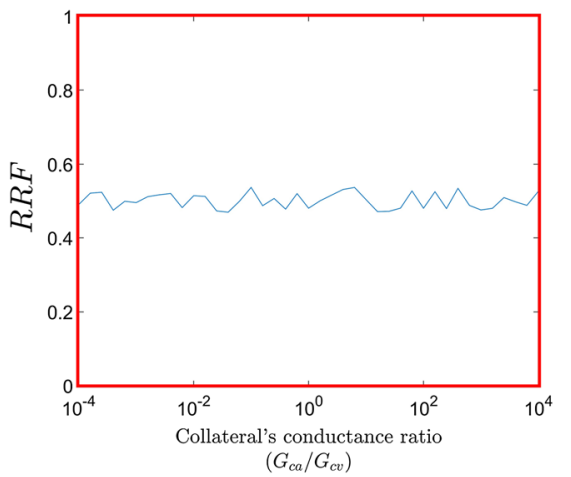

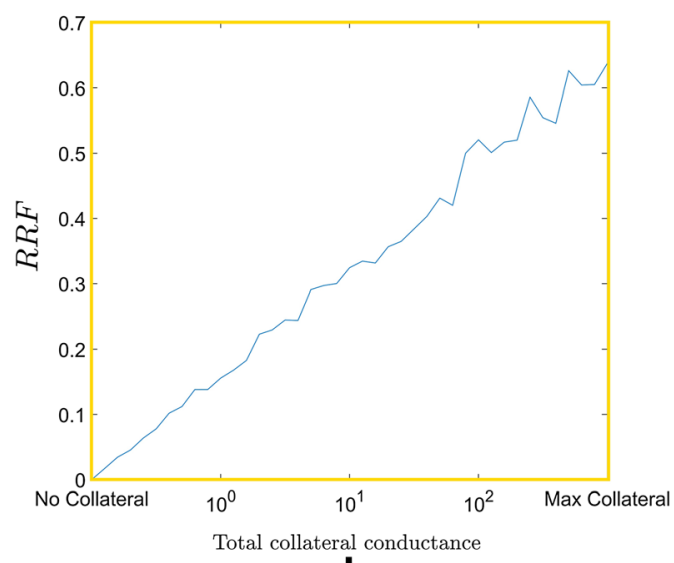

b
Fig. 8 The effects of the balance of arterial and venous collateral conductance (a) and of the total collateral conductance (b) on the relative remaining flow (RRF) in the anastomotic site 4 , demonstrating the loglinear dependence of RRF on total collateral conductance without an

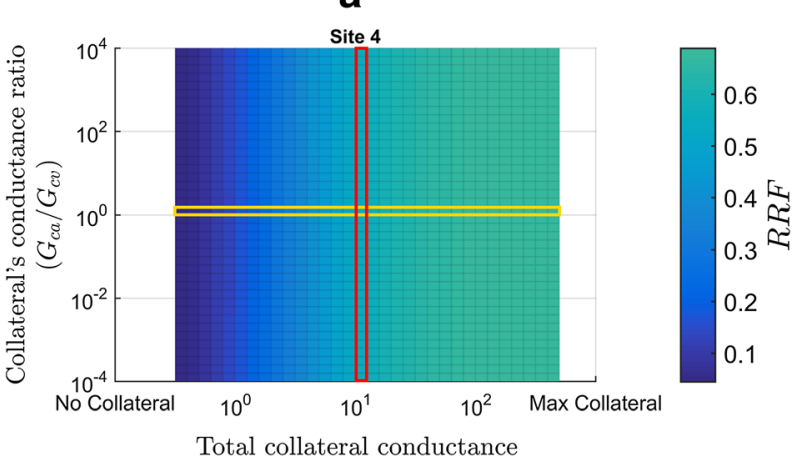

C

effect of arteriovenous balance. RRF for all levels of total collateral conductance and arteriovenous balance (c), demonstrating absence of interactive effects of both parameters 
Open Access This article is distributed under the terms of the Creative Commons Attribution 4.0 International License (http:// creativecommons.org/licenses/by/4.0/), which permits unrestricted use, distribution, and reproduction in any medium, provided you give appropriate credit to the original author(s) and the source, provide a link to the Creative Commons license, and indicate if changes were made.

\section{References}

1. Law S, Fok M, Chu KM, Wong J (1997) Thoracoscopic esophagectomy for esophageal cancer. Surgery 122:8-14. https://doi.org/ 10.1016/S0039-6060(97)90257-9

2. Urschel JD (1994) Esophagogastrostomy anastomotic leaks complicating esophagectomy: a review. Am J Surg 169:634-640

3. Cassivi SD (2004) Leaks, strictures, and necrosis: a review of anastomotic complications following esophagectomy. Semin Thorac Cardiovasc Surg 16:124-132. https://doi.org/10.1053/j.semtcvs. 2004.03.011

4. Zehetner J, DeMeester SR, Alicuben ET, Oh DS, Lipham JC, J a H, DeMeester TR (2014) Intraoperative assessment of perfusion of the gastric graft and correlation with anastomotic leaks after Esophagectomy. Ann Surg 00:1-5. https://oi.org/10.1097/SLA. 0000000000000811

5. Campbell C, Reames MK, Robinson M, Symanowski J, Salo JC (2015) Conduit vascular evaluation is associated with reduction in anastomotic leak after Esophagectomy. J Gastrointest Surg 19:806812. https://doi.org/10.1007/s11605-015-2794-3

6. Kumagai Y, Ishiguro T, Haga N, Kuwabara K, Kawano T, Ishida H (2014) Hemodynamics of the reconstructed gastric tube during esophagectomy: assessment of outcomes with indocyanine green fluorescence. World J Surg 38:138-143. https://doi.org/10.1007/ s00268-013-2237-9

7. Rino Y, Yukawa N, Sato T, Yamamoto N, Tamagawa H, Hasegawa S, Oshima T, Yoshikawa T, Masuda M, Imada T (2014) Visualization of blood supply route to the reconstructed stomach by indocyanine green fluorescence imaging during esophagectomy. BMC Med Imaging 14:18. https://doi.org/10.1186/1471-2342-14-18

8. Koyanagi K, Ozawa S, Oguma J, Kazuno A, Yamazaki Y, Ninomiya Y, Ochiai H, Tachimori Y (2016) Blood flow speed of the gastric conduit assessed by indocyanine green fluorescence. Medicine 95(30):e4386. https://doi.org/10.1097/MD. 0000000000004386

9. Chadi SA, Fingerhut A, Berho M, DeMeester SR, Fleshman JW, Hyman NH, Margolin DA, Martz JE, McLemore EC, Molena D, Newman MI, Rafferty JF, Safar B, Senagore AJ, Zmora O, Wexner SD (2016) Emerging trends in the etiology, prevention, and treatment of gastrointestinal anastomotic leakage. J Gastrointest Surg 20:2035-2051. https://doi.org/10.1007/s11605-016-3255-3

10. Bruinsma P, Arts T, Dankelman J, Spaan JAE (1988) Model of the coronary circulation based on pressure dependence of coronary resistance and compliance. Basic Res Cardiol 83:510-524. https:// doi.org/10.1007/BF01906680

11. Blomley MJK, Coulden R, Bufkin C, Lipton MJ, Dawson P (1993) Contrast bolus dynamic computed-tomography for the measurement of solid-organ perfusion. Investig Radiol 28:S72-S77. https://doi.org/10.1097/00004424-199311001-00023

12. Jansen SM, de Bruin D, Strackee S, van Bavel E, Van LTG, van Berge Henegouwen MI, Gisbertz SS (2018) PS01.186: quantitative perfusion evaluation after gastric tube reconstruction using fluorescence imaging. Dis Esophagus 31:102-103

13. Jacobson ED, Jerry BS (1962) Hemodynamics of the stomach I Resistance-flow Relationship in the Gastric Vascular Bed. Am J Dig Dis 7:779-785
14. Berne RM, Koeppen BM, Stanton BA (2008) Berne \& levy physiology, 6th ed. Mosby/Elsevier, Philadelphia

15. Wiedeman MP (1963) Dimensions of blood vessels from distributing artery to collecting vein. Circ Res 12:375-378. https://doi.org/ 10.1161/01.RES.12.4.375

16. Pfitzner J (1976) Poiseuille and his law. Anaesthesia 31:273-275. https://doi.org/10.1111/j.1365-2044.1976.tb11804.x

17. Meier P, Zierler KL (1954) On the theory of the indicator-dilution method for measurement of blood flow and volume. J Appl Physiol 6:731-744

18. Dormand JRR, Prince PJJ (1980) A family of embedded RungeKutta formulae. J Comput Appl Math 6:19-26. https://doi.org/10. 1016/0771-050X(80)90013-3

19. Schreiner W, Buxbaum PF (1993) Computer-optimization of vascular trees. IEEE Trans Biomed Eng 40:482-491

20. Huyghe JM, Arts T, van Campen DH, Reneman RS (1992) Porous medium finite element model of the beating left ventricle. Am J Phys 262:H1256-H1267

21. Biot MA (1972) Thory of finite deformations of porous solids. Indiana Univ Math J 21:597. https://doi.org/10.1512/iumj.1972.21.21048

22. Kresh JY, Fox M, Brockman SK, Noordergraaf A (1990) Modelbased analysis of transmural vessel impedance and myocardial circulation dynamics. Am J Phys 258:H262-H276

23. May-newman K, Mcculloch AD (1998) Homogenization modeling for the mechanics of perfused myocardium. Prog Biophys Mol Biol 69:463-481

24. Ishiguro T, Kumagai Y, Ono T, Imaizumi H, Honjo H, Suzuki O, Ito T, Haga N, Kuwabara K, Sobajima J, Kumamoto K, Ishibashi K, Baba H, Ishida H, Kawano T (2012) Usefulness of indocyanine green angiography for evaluation of blood supply in a reconstructed gastric tube during esophagectomy. Int Surg 97:340-344. https:// doi.org/10.9738/cc159.1

25. Phillips BT, Lanier ST, Conkling N, Wang ED, Dagum AB, Ganz JC, Khan SU, Bui DT (2012) Intraoperative perfusion techniques can accurately predict mastectomy skin flap necrosis in breast reconstruction. Plast Reconstr Surg 129:778e-788e. https://doi.org/ 10.1097/PRS.0b013e31824a2ae8

26. Komorowska-Timek E, Gurtner GC (2010) Intraoperative perfusion mapping with laser-AssistedIndocyanine green imaging can predict and PreventComplications in immediate breast reconstruction. Plast Reconstr Surg 125:1065-1073. https://doi.org/10.1097/ PRS.0b013e3181d 17f80

27. Pacheco PE, Hill SM, Henriques SM, Paulsen JK, Anderson RC (2013) The novel use of intraoperative laser-induced fluorescence of indocyanine green tissue angiography for evaluation of the gastric conduit in esophageal reconstructive surgery. Am J Surg 205: 349-353. https://doi.org/10.1016/j.amjsurg.2012.11.005

28. Landau MJ, Gould DJ, Patel KM (2016) Advances in fluorescentimage guided surgery. Ann Transl Med 4:392. https://doi.org/10. 21037/atm.2016.10.70

29. Yukaya T, Saeki H, Kasagi Y, Nakashima Y, Ando K, Imamura Y, Ohgaki K, Oki E, Morita M, Maehara Y (2015) Indocyanine green fluorescence angiography for quantitative evaluation of gastric tube perfusion in patients undergoing Esophagectomy. $\mathrm{J}$ Am Coll Surg 221:e37-e42. https://doi.org/10.1016/j. jamcollsurg.2015.04.022

30. Kamiya K, Unno N (2015) Quantitative assessment of the free jejunal graft perfusion. J Surg Res 194:394-399. https://doi.org/ 10.1016/j.jss.2014.10.049

Publisher's note Springer Nature remains neutral with regard to jurisdictional claims in published maps and institutional affiliations. 


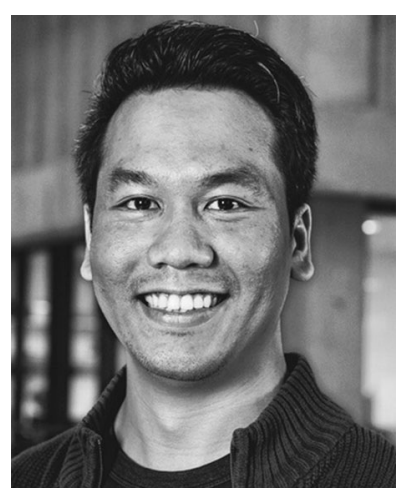

Haryadi Prasetya is a $\mathrm{PhD}$ candidate in the Department of Biomedical Engineering \& Physics, Amsterdam University of Medical Centers, the Netherlands. He was a research assistant in Biomedical Engineering Lab, Bandung Institute of Technology, Bandung, Indonesia. Currently he focuses on computational modeling and quantitative analysis of tissue perfusion.

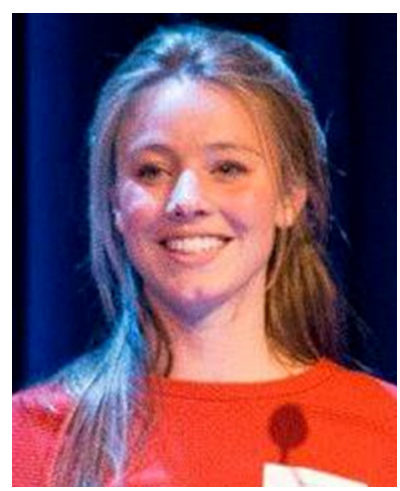

Sanne Jansen graduated as MD in 2014 at the University of Groningen, the Netherlands and is a $\mathrm{PhD}$ candidate in the Department of Biomedical Engineering \& Physics and the Department of Surgery, Amsterdam University of Medical Centers, the Netherlands. Currently she is a Radiology resident in the Department of Radiology, OLVG, Amsterdam and will defend her thesis on quantitative perfusion diagnostics in esophageal cancer surgery at the end of 2018 .

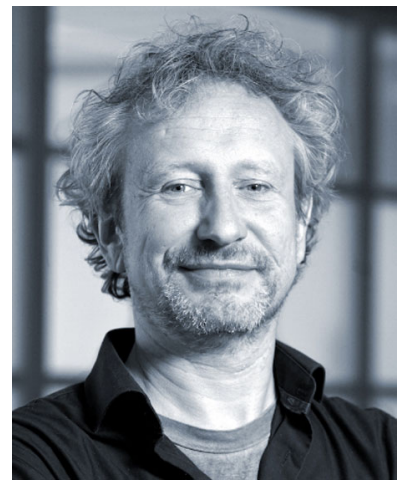

Henk Marquering obtained his $\mathrm{PhD}$ at the Geophysics department of the Utrecht University, the Netherlands in 1996. After a post-doc in Princeton University, USA, he changed careers to work at the R\&D department of Océ, currently Canon, where he worked on document image analysis. He switched between academia and industry a couple of times after which he moved to the Amsterdam UMC, departments Radiology \& Nuclear Medicine and Biomedical Engineering and Physics. He is currently associate professor focusing on cardiovascular imaging and image processing. He is also a co-founder of the AMC spinoff Nico.lab in which AI techniques are used to support diagnosis of stroke patients.

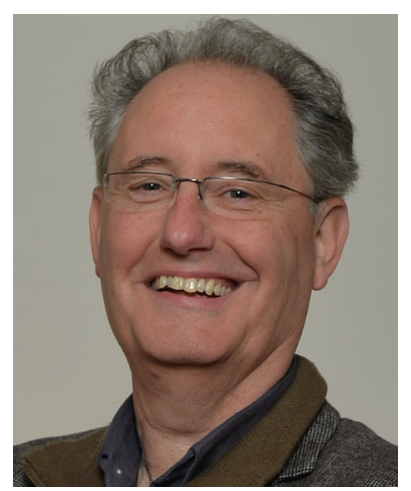

Ton van Leeuwen graduated in physics (with honors) at the University of Amsterdam in 1989. He obtained his $\mathrm{PhD}$ (with honors) at the University of Utrecht based on his work on pulsed laser ablation of tissue at the Laboratory for Experimental Cardiology at the UMC Utrecht. After a post-doc period and an ICIN fellowship at CWRU (Cleveland, Ohio), he became in 1998 staff member of the Laser Center at the Academic Medical Center of the University of Amsterdam (AMC), the Netherlands. In 2001, he was appointed as professor in Clinical Application of Biomedical Optics at the University of Twente, at which he headed the Biomedical Optics group from 2003 to 2008. In 2008 he was appointed as professor in Biomedical Photonics and head of the new BME \& Physics department at the AMC, followed by the appointed as full professor in Biomedical Physics in 2009. Current research focuses on the physics of the interaction of light with tissue, and to use that knowledge for the development, introduction and clinical evaluation of (newly developed) optical imaging techniques for gathering quantitative functional and molecular information of tissue. Ton van Leeuwen is fellow of the AIMB and SPIE.

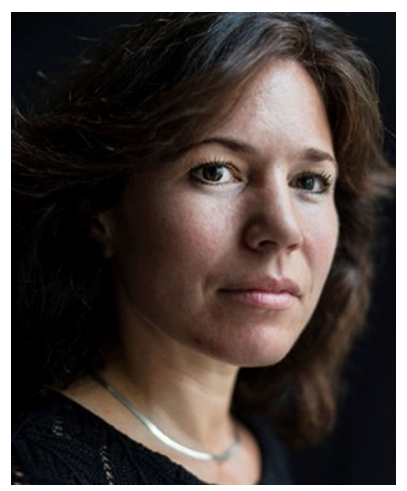

Suzanne Gisbertz finished medical school at the University of Amsterdam in 2002. She did her surgical training at Utrecht University until 2010 and her advanced gastro-intestinal and minimally invasive surgical training at VU medical center Amsterdam (professor Miguel Cuesta) until 2012. She is currently a senior staff member of the upper-GI unit at the Academic Medical Center in Amsterdam. She is a trainer in minimally invasive gastrectomies and esophagectomies at different Dutch and international centers. Her main research topics are minimally invasive upper GI surgery and postoperative outcome, extent of lymphadenectomy in esophageal and gastric cancer. 


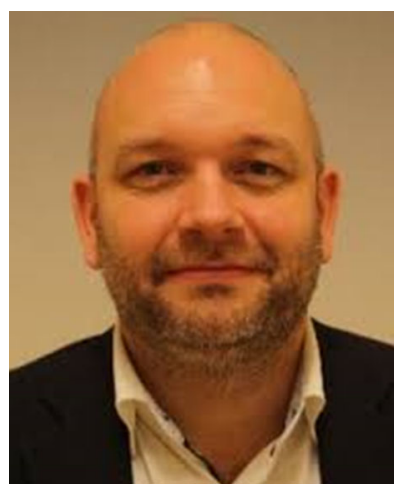

Daniel M. de Bruin finished his PhD at the Biomedical Engineering \& Physics department (BMEP) at the Academic Medical Center of the University of Amsterdam (AMC-UvA). He is a staff member, performing translational research at both the department of Urology and the photonics group of BMEP of the AMC-UvA. His projects revolve around digital pathology using convolutional neural networks as computer aided diagnosis for pathologists, optical coherence tomography, con-focal laser endomicroscopy, and irreversible electroporation.

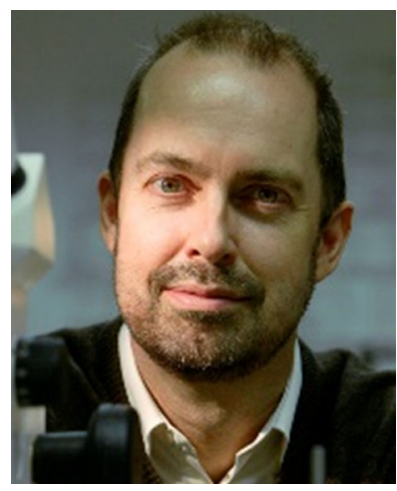

Ed van Bavel obtained his $\mathrm{PhD}$ cum laude from the University of Leiden in 1989. He then moved to the department of Medical Physics of the Academic Medical Centre (AMC-UvA), where he was post-doc, assistant and associate professor. He now is a full professor of vascular biophysics at the department of Biomedical Engineering and Physics of the Amsterdam University Medical Centers. He specializes in vascular biophysics, biomechanics, cardiovascular and neurovascular biology and physiology, (co) authoring 256 publications in these fields. He has been member of several editorial boards and councils, was the president of the European Society for Microcirculation and organized the European Conference on Microcirculation in 2006. 